\title{
Design and Experiment of Online Pesticide Mixing System Based on PWM
}

\author{
Du Yansheng1, a, Li Chengzhen 2,b , Chen Zhigang $^{*, 1,3, c}$, Wei Xinhua ${ }^{3, d}$ \\ ${ }^{1}$ School of The Environment and Safety Engineering, Jiangsu University, Zhenjiang, 212013, China \\ 2 School of Electronic and Information Engineering,Jiangsu University,Zhenjiang 212013,China \\ ${ }^{3}$ Key Laboratory of Modern Agricultural Equipment and Technology,Ministry of Education, \\ Jiangsu University, Zhenjiang, 212013, China \\ a dys04@163.com, b 1285859957@qq.com, *cchenzg01@126.com, dwei_xh@126.com \\ ${ }^{*}$ Corresponding author: Chen Zhigang,, chenzg01@126.com
}

\begin{abstract}
Keywords: Online pesticide mixing, PWM, Jet-mixing , Mixing ratio
Abstract. In order to improve the mixing ratio of online pesticide mixing system, and control the flow of pesticide in the system. Jet-mixing method was adopted, Structure parameters and material of Jet-mixing device were optimal selected, online pesticide mixing system based on PWM variable was designed and built, PWM was used to control the flow of pesticide in the system. Pesticide flow control system based on MCU was designed. For the whole mixing system, the experiment was carried out. As a result, with the pesticide flow control system, mixture ratio of online pesticide mixing system can be improved generally. Under the same frequency, with duty cycle of PWM signals decreased, mixing ratio increased, when the frequency reached $20 \mathrm{~Hz}$ and duty cycle become $1 / 10$, mixing ratio reaches 193.84 , the effect of pesticide flow controlling was best, compared without the control system, mixing ratio was increased by nearly 20 times.
\end{abstract}

\section{Introduction}

For the traditional pre-mixing pesticide, the farmers need contact with pesticide and this behavior has certain harm to the farmers. What's more, it is difficult to control the dosage of the mixing pesticide, and the excess pesticide will produce certain pollution to the environment ${ }^{[1 \sim 3]}$. Compared with the traditional pre-mixing pesticide, online-mixing method saves pesticide, reduces the pollution of pesticide to the environment, reduces the harm of pesticide to the farmers and improves the safety of mixing pesticide ${ }^{[4-5]}$. All the time many experts and scholars pay their attention to the research on the online-mixing pesticide and pulse width modulation (PWM) technology. Baijing Qiu et al made experiment on the influence of the jet-mixing apparatus on working state of the online mixing spray system. And he studied on the effect about different structure parameters of jet-mixing apparatus, but the spray system can't control the dosage of the pesticide drawn by jet-mixing apparatus ${ }^{[6-8]}$. Giles built the "head + solenoid valve" model that a nozzle and solenoid valve connected directly in his study ${ }^{[9]}$. Xinhua Wei et al built a solenoid valve combined nozzle's spray flow model which combined the speed of sprayer. But the PWM was used to control the spray flow instead of dosage of pesticide ${ }^{[10]}$. Junfeng Zhang et al established proportional control platform of online jet-mixing pesticide, but the increased range of the mixing ratio is finite ${ }^{[11]}$.

In this paper, PWM is used to control the pesticide flow, the pesticide flow control system based on MCS-51 single chip is designed. Online jet-mixing experiment with the different duty cycle and frequency of PWM signals from the single chip is carried out, and the effect of controlling the mixing ratio in the control system is studied.

\section{Online pesticide mixing system based on PWM}

The structure of online mixing system is shown in Figure 1, the system is composed of water tank, pesticide tank, triplex plunger pump, high-speed solenoid valve, micro-liquid flow sensor and turbine flow sensor. The length of spray rod is 8 meters, 20 nozzles are installed in the spray rod at equal 
distance. The distance between the two nozzles is $40 \mathrm{~cm}$. The model of the nozzle is F110-015(Suzhou Landor Precision Mechanics Co., Ltd.).

When the system works, water is pumped by the triplex plunger pump to the inlet of the jet-mixing apparatus, the high-speed flow is injected in the inhaled chamber of the apparatus and the negative pressure is generated in the apparatus. The simulated pesticide (simulated pesticide is mixed with dye and water in special proportion, hereafter this simulated pesticide will be named simply as pesticide) is also sucked in the apparatus. Water and pesticide are mixed automatically in the mixing chamber of the apparatus. And the mixed liquors will be injected in the spray rod. The PWM signals with different duty cycle and frequency are output by the controller based on single chip to control the opening and closing of the high-speed solenoid valve which is set in the pipeline inhale pesticide, thereby controlling the flow of pesticide and reaching the purpose of controlling the mixing ratio of the system. The micro-liquid flow sensor tests the flow of pesticide and display instantaneous and cumulative flow on flow totalizer in real time.

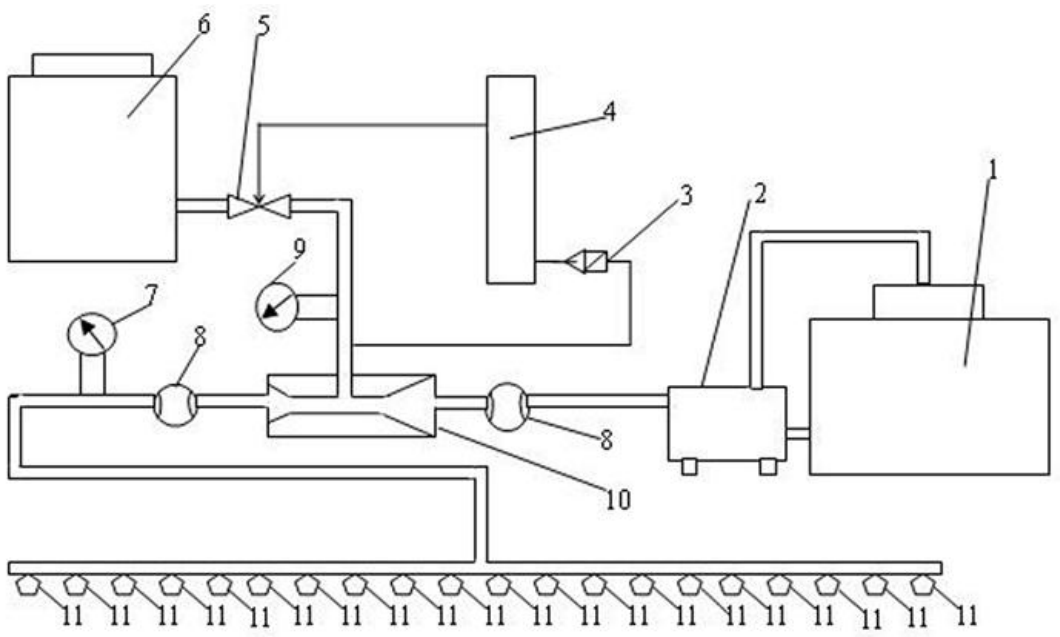

Figure 1. Sketch map of the online mixing pesticide system

1.Water tank, 2.Triplex plunger pump, 3.Micro-liquid flow sensor, 4.Single chip, 5.High-speed solenoid valve, 6.Pesticide tank, 7.Pressure gauge, 8.Turbine flow sensor, 9.Vacuum pressure gauge, 10.Jet-mixing apparatus, 11.Nozzle.

\section{Jet-mixing apparatus}

According to the study about online spray system's mixing performance of Baijing Qiu [8], combining with the system's requirement about spray flow, pressure and so on, the related structural parameters of jet-mixing apparatus are selected optimally ${ }^{[12 \sim 16]}$. The diameter of mixing chamber is $4 \mathrm{~mm}$, the outlet diameter of jet nozzle is $2 \mathrm{~mm}$ and the material of the apparatus is stainless steel. Apparatus's structure is shown in Figure 2 and the related parameters are shown in Table 1.

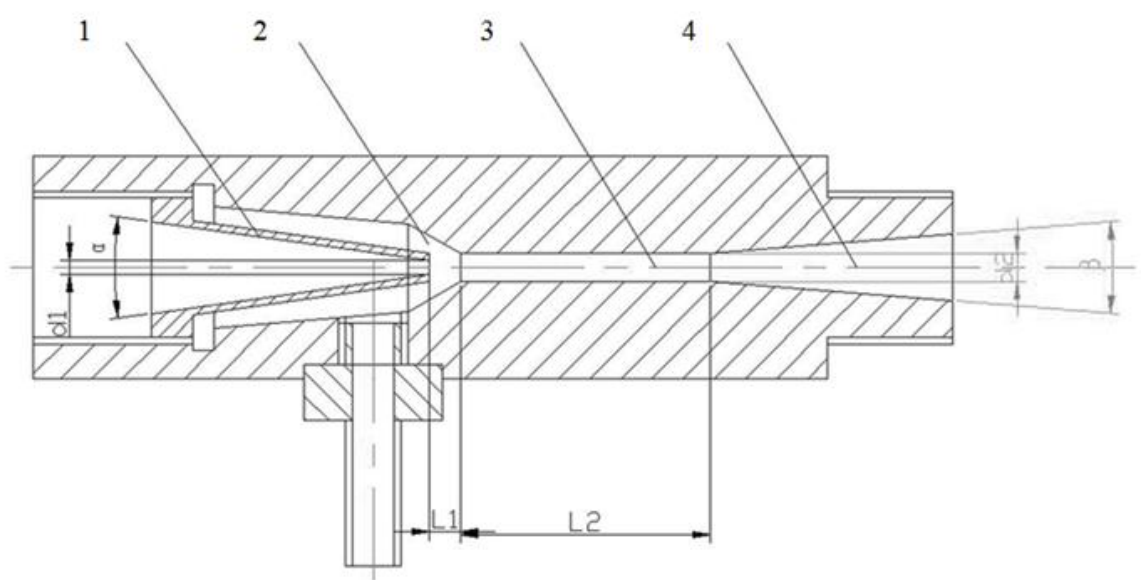

Figure 2. Jet-mixing apparatus

1. Jet nozzle, 2.Inhaled chamber, 3.Mixing pipeline, 4.Diffusion pipeline. 
Table 1. Structural parameters of jet-mixing apparatus

\begin{tabular}{|c|c|}
\hline Constringent angle of jet nozzle $\alpha\left({ }^{\circ}\right)$ & 16 \\
\hline Outlet diameter of jet nozzle d1 $(\mathrm{mm})$ & 2 \\
\hline Distance between jet nozzle and mixing pipeline L1 $(\mathrm{mm})$ & 4.5 \\
\hline Length of mixing pipeline L2 $(\mathrm{mm})$ & 36 \\
\hline Diffusion angle of diffusion pipeline $\beta\left({ }^{\circ}\right)$ & 4 \\
\hline Diameter of mixing pipeline d2 $(\mathrm{mm})$ & 4 \\
\hline
\end{tabular}

\section{Pesticide flow control system}

In order to control of the mixing ratio of the online mixing pesticide system, the pesticide flow control system based on the single chip is designed. The structure principle of the system is shown in figure 3. The system is mainly composed of button module, LED display module, high-speed solenoid valve and its driving circuit, flow sensor and so on.

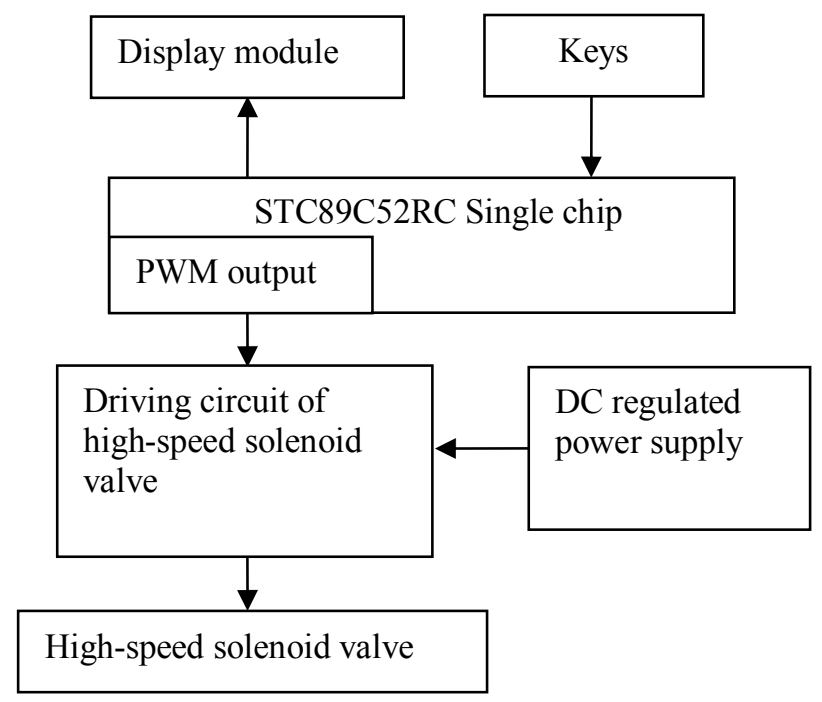

Figure 3. Pesticide flow control system

The model of the single chip is STC89C52RC, the single chip has the characteristic of high speed, low power consumption, strong anti-interference and so on. In this system, the single chip is used to gather and dispose the key signal, display signal, PWM signal and so on. The duty cycle and frequency of PWM can be changed by the two separated key, and the number of the duty cycle and frequency will be displayed on display module composed of LED.

High-speed solenoid valve's model is 6013 direct acting solenoid valve (Burkert in German). Power supply voltage: $24 \mathrm{~V}$, DC, working pressure: $0 \sim 0.6 \mathrm{MPa}$, maximum flow rate: $5 \mathrm{~L} / \mathrm{min}$, opening time: $20 \mathrm{~ms}$, closing time: $30 \mathrm{~ms}$, interface size: $3 / 8$ internal thread.

Turbine flow sensor measures the total flow of the water and pesticide, it's model is LWGY-MK-DN6 (Hangzhou Mecco Sensing Technology Co., Ltd.), the range: 0.1 0.6 m3/h, accuracy: $\pm 2 \% \mathrm{~F} . \mathrm{S}$, output signal:4 20mA current, voltage: $24 \mathrm{~V}$, DC.

Micro flow sensor measures the flow of pesticide, it's model is 931 series (Beijing Tide Science Technology Co., Ltd.), the range: $0.025 \sim 12 \mathrm{~L} / \mathrm{min}$, accuracy: $\pm 2 \% \mathrm{~F} . \mathrm{S}$, output signal: pulse output (NPN), voltage: $4.5 \sim 24 \mathrm{~V}$, DC.

\section{Experiment conditions}

In order to reflect the effect of control system, comparative test that without the controlling of pesticide control system is designed. The working pressure is set to $0.3 \mathrm{MPa}$ when the F110-015 nozzle can work well. The test lasts 10 minutes, the mixing ratio (water : pesticide) is 11.03:1. 
Then the pesticide control system is enabled, the working pressure is also set to $0.3 \mathrm{MPa}$. The duty cycle of PWM will be set range of 1/10 1, step: 1/10, and frequency will be set as $1 \mathrm{~Hz}, 2 \mathrm{~Hz}$, $5 \mathrm{~Hz}, 8 \mathrm{~Hz}, 10 \mathrm{~Hz}, 16 \mathrm{~Hz}, 20 \mathrm{~Hz}$.

\section{Results and discussion}

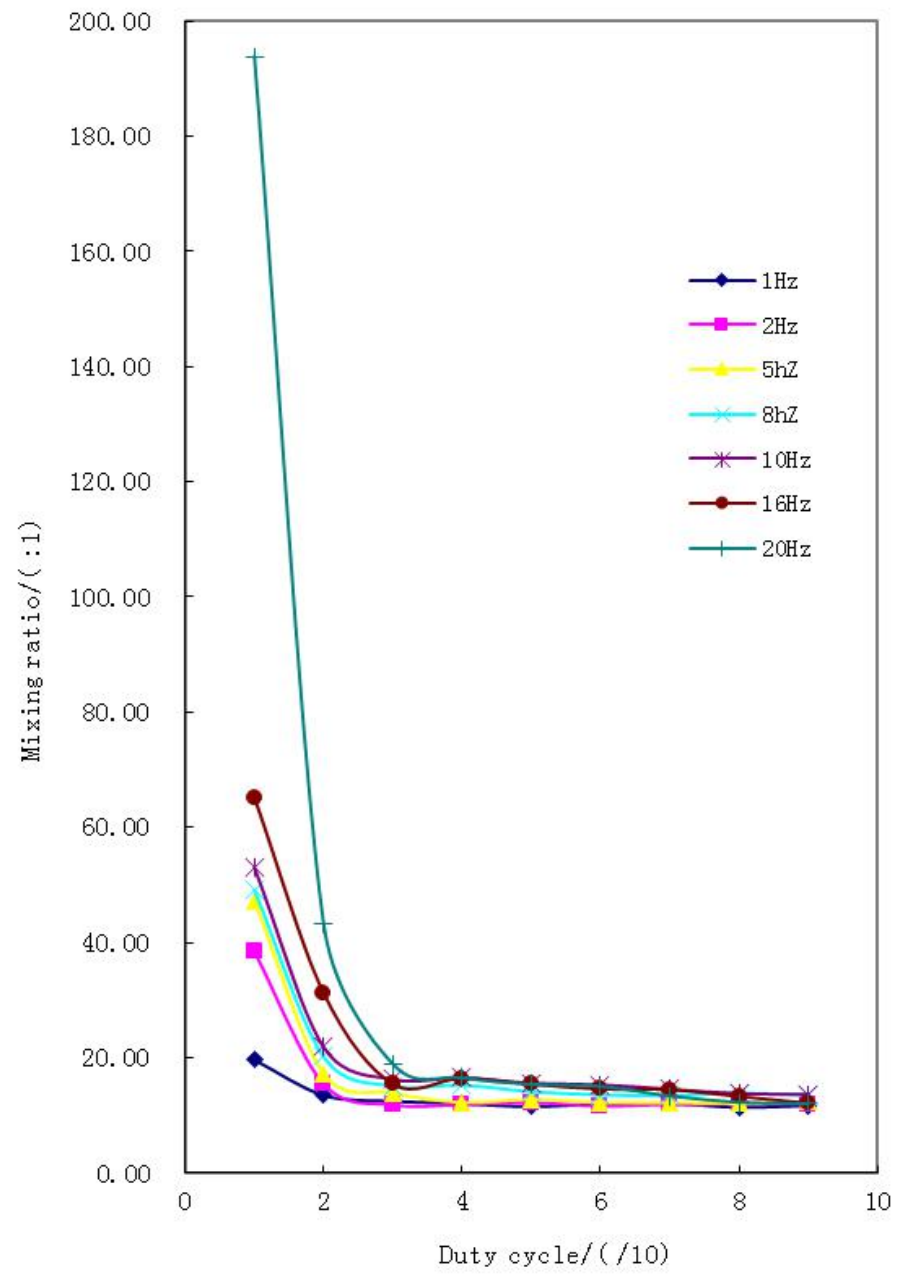

Figure 4. Mixing ratio with different duty cycle and frequency of online mixing system

From the result of comparative test, the mixing ratio is $11.03: 1$ without the pesticide flow control system. Figure 4shows that the mixing ratio is improved generally when the control system works. When the duty cycle is $1(10 / 10)$, the solenoid valve is always open, so the mixing ratio is close to the result of comparative test. At the same frequency, the mixing ratio increase with the duty cycle of PWM decreases. When the frequency reached $20 \mathrm{~Hz}$ and the duty cycle become $1 / 10$, the mixing ratio reaches 193.84, the effect of pesticide flow controlling is best, compared without the control system, mixing ratio was increased by nearly 20 times.

\section{Conclusions}

Online pesticide mixing system based on PWM and the pesticide flow control system based on single chip are designed. In the control system, the frequency and duty cycle of PWM can be changed by two separated keys, and the number of the duty cycle and frequency will be displayed on display module composed of LED. The systems with low cost are simple, practical.

The pesticide flow control system based on single chip makes the mixing ratio of online mixing system adjustable during $11 \sim 190$.

When the pesticide flow control system works, the mixing ratio is improved generally. Especially, the frequency reached $20 \mathrm{~Hz}$ and the duty cycle become $1 / 10$, the mixing ratio reaches 193.84 , the 
effect of pesticide flow controlling is best, compared without the control system, mixing ratio is increased by nearly 20 times.

\section{Acknowledgements}

This work was financially supported by the Jiangsu provincial science and technology support program (BE2014415), and the agricultural science and technology support program of Zhenjiang (NY2014030), and the Natural Science Research Project of Higher Education of Jiangsu Province, China under Grant(14KJB610004).

\section{References}

[1] Yanxuan Liu, Huidong Bai, Guiying Jiang. Chinese Agricultural Science Bulletin,2007,23 (7) : 577 582. (in Chinese)

[2] Yong Chen, Jiaqiang Zheng. Transactions of the CSAE, 2005, 21(5): 69 72. (in Chinese)

[3] Xinhua Wei, et al. Journal of agricultural mechanization research, 2011(2): 9 14. (in Chinese)

[4] Xiongkui He. Transactions of the Chinese Society of Agricultural Engineering, 2004, 20(1): 13 15. ( in Chinese)

[5] Zetian Fu, Lijun Qi, Junhong Wang. Transactions of the Chinese Society for Agricultural Machinery, 2007, 38( 1) : 189 192. ( in Chinese)

[6] Shuren Chen, Dongfu Yin, Xinhua Wei,et al. Journal of Drainage and Irrigation Machinery Engineering,2011,29( 3) : 272 276. (in Chinese)

[7] Changyuan Zhai, Ruixiang Zhu, Sheng Huang,et al. Transactions of the Chinese Society for Agricultural Machinery,2011,42(8):70 74. ( in Chinese)

[8] Baijing Qiu, Jing Ma, Bin Deng, et al . Transactions of the Chinese Society of Agricultural Engineering (Transactions of the CSAE), 2014, 30(17): 78 85. (in Chinese)

[9] Giles D K, Comino J A. Journal of Agricultural Engineering Research, 1990, 47(4): 249 267.

[10] Xinhua Wei, Shan Jiang, Jinmin Zhang,et al. Transactions of the Chinese Society for Agricultural Machinery,2013,44(2) : 87 92. ( in Chinese)

[11] Weidong Jia, Zhigang Chen, Xin Zhao,et al. Transactions of the Chinese Society for Agricultural Machinery,2013,44(8) : 90 93. (in Chines) 